\title{
Understanding the Effect of Processing on the Structure of Plant Cell Walls as a Mean to Design Novel Clean Label Ingredients
}

\author{
Joël Wallecan ${ }^{1}$, Jinping Dong ${ }^{2}$, Katlijn Moelants ${ }^{1}$, Tristan Lipkie ${ }^{2}$, Adam Steinbach $^{2}$, Jacques Mazoyer $^{3}$ \\ 1. Ingredient, Material \& Nutrition R\&D, Cargill R\&D Centre Europe, Vilvoorde, Belgium \\ 2. Ingredient, Material \& Nutrition R\&D, Cargill Minneapolis R\&D Centre, Plymouth, USA \\ 3. Centre of Expertise Texture, Cargill Starches Sweeteners \& Texturizers, Carentan, France
}

Plant cell walls are complex assemblies consisting of cellulose, hemicellulose, pectin, proteins, lignin... These composite materials are synthesized by the plant in order to provide strength, shape, growth, integrity and protection to the cells. Food processes on the other hand aim at disrupting these tight networks in order to extract the high-value ingredients inside the cell and cell wall: oil, starch, proteins, pectin, carrageenan, vitamins... This leads to the generation of 2 main streams: a high-value ingredient stream (e.g. pectin) and a disrupted plant cell wall side stream. Until recently, limited attention was paid to these side streams and they were often poorly valorized or even discarded without added value. However, these plant cell wall streams have gained accrued interest over the last decade for various reasons: economical, sustainability, consumer behavior, cost of raw materials...

Compared to commonly-used hydrocolloid ingredients (e.g. pectin, carrageenan, starch...), the structureforming properties of food grade plant cell wall-derived fibers currently on the market (citrus fibers, wheat bran, corn fibers, bamboo fibers, soy fibers, sugar beet fibers...) are fairly limited which limits their valorization pathways. The latter are often poorly hydrating, sediment rapidly and thereby present limited texture properties. Mechanical processing (i.e. using high-pressure homogenization) is known to alter the physicochemical properties of plant-based fiber suspensions. During this step, particle sizes and morphologies are reduced to a certain micro- or even nano-scale level through mechanisms of fragmentation and defibrillation (Figure 1a). The extent of de-structuring largely depends on the amount of energy input as well as on the raw material composition. This results in enhanced functional properties such as water holding capacity (WHC) (Figure 1b), extent of swelling, oil binding capacity (OBC), antioxidant capacity and the cation exchange capacity (CEC) [1-2].

In order to explain the changes at bulk level a thorough understanding of the microstructural evolutions as function of processing is required. A range of techniques is usually required in order to enable one to visualize the various length scales involved in the build-up of the plant cell wall architecture. The obtained data provides a wealth of information to food scientists as it enables them to tailor the various processing steps to the plant cell wall composition and architecture. Elements such as type and amount of pectin, as well as its location in the cell wall enable to drive the key processing steps required to defibrillate citrus fibers. The defibrillation conditions will in turn affect the microstructure of the obtained citrus fiber suspension and as such its bulk properties. Significant increases in the water holding and swelling capacity, rheology and the emulsion stabilization properties due to mechanical processing were for example found for various insoluble fiber fraction of carrot pomace [3] tomato paste [4] or orange pulp [5-6].

Additionally, quantitative data can be extracted from microstructural characterizations by using adequate labeling and image analysis techniques. These data can then be used in correlation with the bulk properties 
in order to better approach the mechanistic understanding of how these plant cell walls develop functional properties and behave in food applications.

\section{References:}

[1] Debon, S.J.J., Wallecan, J. and Mazoyer, J., Applied Rheology 22 (2012), p. 63919.

[2] Hu, J.-L., Nie, S.-P. and Xie, M.-Y., Food Chemistry 138 (2013), p. 2338.

[3] Chau, C., Wang, Y. and Wen, Y., Food Chemistry 100 (2007), p. 1402.

[4] Bengtsson, H. and Tornberg, E., Journal of Texture Studies 42 (2011), p. 268.

[5] Van Buggenhout, et al, Innovative Food Science and Emerging Technologies 30 (2015), p. 51.

[6] Wallecan, J., et al, Food Hydrocolloids 47 (2015), p. 115.
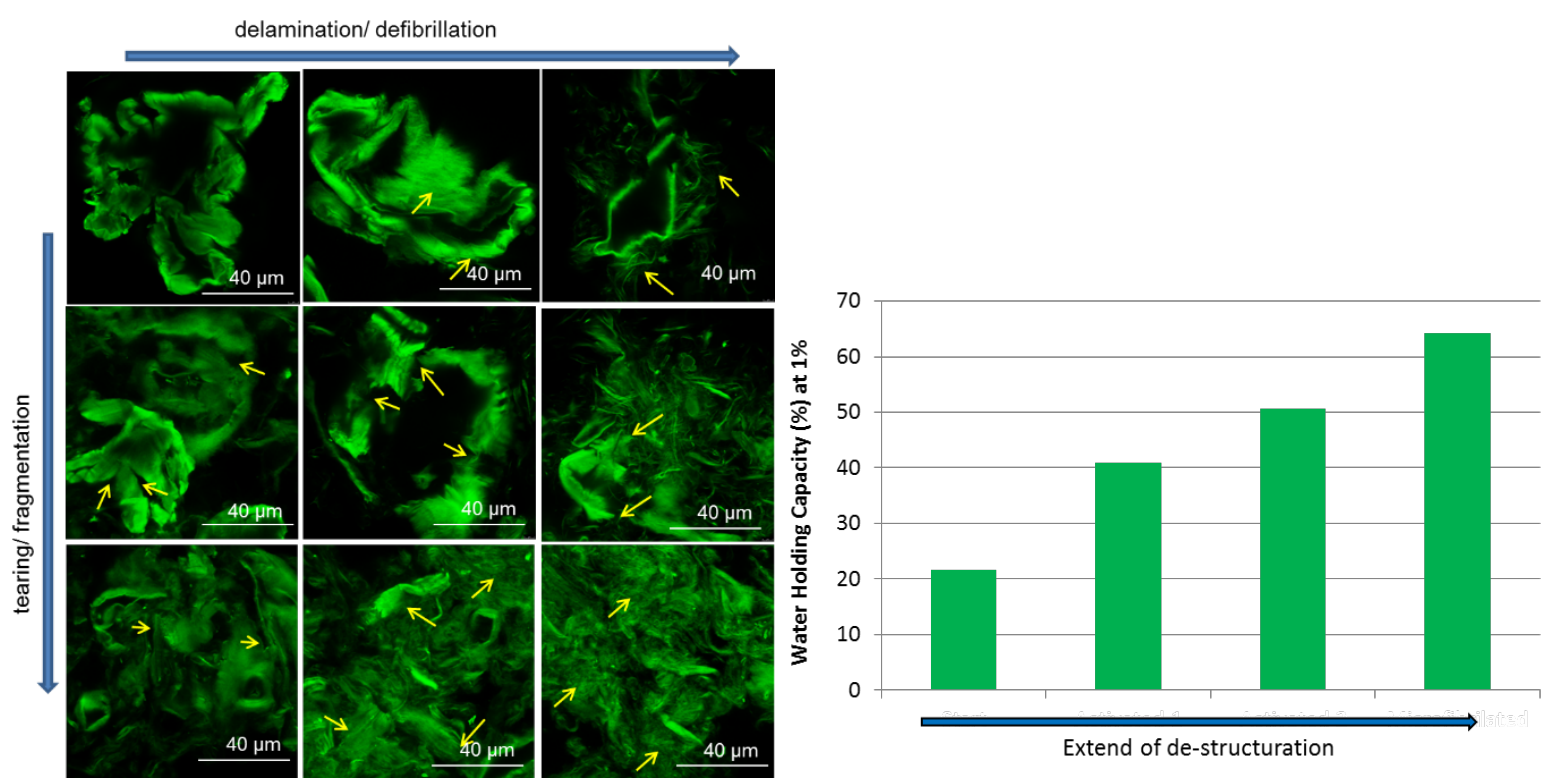

Figure 1. evolution of the microstructure of a citrus fiber sample subjected to various high-pressure homogenization conditions (a) and the corresponding evolution of the water holding capacity of the generated suspensions (b). 